\title{
Static-junction craniospinal irradiation: verification of daily dose and long-term treatment results
}

\author{
Aleksandra Napieralska', Michał Radwan², Iwona Brąclik², Sławomir Blamek
}

\begin{abstract}
${ }^{1}$ Radiotherapy Department, Maria Sklodowska-Curie National Research Institute of Oncology, Gliwice Branch, Gliwice, Poland 2Radiotherapy Planning Department, Maria Sklodowska-Curie National Research Institute of Oncology, Gliwice Branch, Gliwice, Poland
\end{abstract}

Introduction. The study was performed to evaluate the repeatability and effectiveness of the static-junctions image guided (SJIG) method of craniospinal irradiation.

Material and methods. An analysis of 40 treatment plans was performed. All treatment plans were reviewed with regard to the distances between isocentres between in every single field of each fraction during all treatment days. Based on that data, second (actually treated) plans were created. The planned and treated parameters were compared.

Results. The study group consisted of 40 patients irradiated in the craniospinal region. Data on 902 fractions and 1635 isocentres positions was collected. 1-, 2- and 5-year overall survival was 95\%, 89\% and 78\%, respectively. Spine metastases were observed in regions which were covered with a homogenous dose during treatment.

Conclusions. SJIG is safe and produces very good long-term outcomes. Treatment planning and delivery is simple with good reproduction of the planned dose distribution during the actual treatment.

Key words: medulloblastoma, craniospinal irradiation, image guided radiotherapy, treatment planning

\section{Introduction}

Craniospinal irradiation (CSI) is commonly used in the treatment of patients with primary central nervous system tumours which spread via cerebrospinal fluid [1, 2]. CSI is a very challenging technique due to the length and the complexity of the planning target volume (PTV) and radiosensitivity of the surrounding organs. Different approaches to this treatment technique have been developed so far, but no clear advantage of any of them is so far evident [3-18]. The analysis by SIOPE-BTG showed the benefit of modern radiotherapy techniques (intensity modulated radiotherapy - IMRT, volumetric arc therapy - VMAT or proton beam therapy - PBT), but standard (unmodulated) techniques of conformal therapy are still widely used in many treatment centres, especially in low-income countries [6-8].

In this study we evaluated the reproducibility and the effectiveness of the static-junctions image guided (SJIG) method of craniospinal irradiation (CSI) with an emphasis on actual treatment delivery implementation, long-term treatment results and patterns of progression. This method of CSI could be useful in low-income countries with a lack of more advanced treatment techniques and also in countries in which proton

\section{How to cite:}

Napieralska A, Radwan M, Brąclik I, Blamek S. Static-junction craniospinal irradiation: verification of daily dose and long-term treatment results. NOWOTWORY J Oncol 2021; 71:343-347. 
beam treatment is not available. It could also be considered a paediatric treatment technique because the impact of a low dose was associated with dynamic techniques and an integral dose is still a matter of debate.

\section{Material and methods}

\section{Immobilization, imaging and treatment planning}

A thermoplastic mask (Orfit by Stanley) was made individually for each patient. A computed tomography (CT) covering the whole spinal axis was performed with 3-5 $\mathrm{mm}$ slices. A fusion of the CT with a magnetic resonance of the brain was carried out for all the patients. Forty patients were treated, 35 in the supine position and 5 in the prone position.

All the plans were created in the Eclipse Advanced Treatment Planning Software from Varian Medical Systems with the pencil beam convolution (Eclipse PBC) or the analytical anisotropic algorithm (Eclipse AAA). All the retrospectively reviewed plans were created with the AA algorithm version 8.6. Each patient was irradiated with a 3D image-guided technique with static junctions between the fields (SJIG) - the field dimensions and isocenter positions were constant throughout the whole treatment.

The target volume consisted of GTV (gross tumour volume) after the subtotal resection in all cases. The clinical target volume (CTV) was defined as the intracranial content and thecal sac, including nerve roots. The PTV (planning target volume) was created by adding a $5 \mathrm{~mm}$ margin to the CTV. The dose was prescribed to obtain $>95 \%$ of the prescribed dose in $>98 \%$ of PTV.

For all the patients, a single treatment plan with static junctions between the fields was made. It consisted of 2-3 isocentres and 3-7 fields: two opposed lateral fields to treat the brain and a part of the cervical spine, and one or two adjoining posterior spinal fields to cover the rest of the spinal canal (the last one was tilted to match the beam divergence of the main spinal field). The first isocentre was located at the level of the cranial base, and treatment fields covered the brain and the upper part of the cervical spine. The second isocentre was located in the lower part of the thoracic spine (one posterior field, 180 degrees). The third was used in the case of taller patients or when the thoracic field did not acceptably cover the anterior part of the lumbosacral space. The number of spinal fields (and isocentres) depended strictly on the length of the PTV due to the limited maximum field size in the Varian system which is (with SSD of $100 \mathrm{~cm}$ ) $40 \times 40 \mathrm{~cm}$ at the isocentre. All the isocentres had the same vertical and lateral coordinates. Corrections in longitudinal direction during treatment were allowed only in the first isocentre position, corrections in lateral and vertical direction were allowed in all isocentres positions. The position of treatment fields between the first and the second isocentre (the first junction) was matched using the rotation of the collimator and treatment couch. The second junction (if necessary) was created using the treatment table rotation (90 degrees) and adjustment of the gantry rotation (fig. 1). This method eliminates overlapping or underdosage between the fields. All the treatment plans had dosimetric verification before the beginning of treatment.

\section{Treatment process}

All the patients were treated on standard linear accelerators with energies of 6-20 MV.Verification of the patient's position was performed with the portal view images (PVI) - the earlier years of the study or with kilovoltage imaging (kV). To assure correct treatment delivery, the couch was moved in a longitudinal axis by a constant value derived from the treatment plan, all other shifts were corrected according to the results of imaging on the treatment machine.

\section{Review process}

All the treatment plans were reviewed by the physician and the physicist with regard to the treatment couch movement (and the distance between isocentres) between every single field of each fraction during all the treatment days, based on the
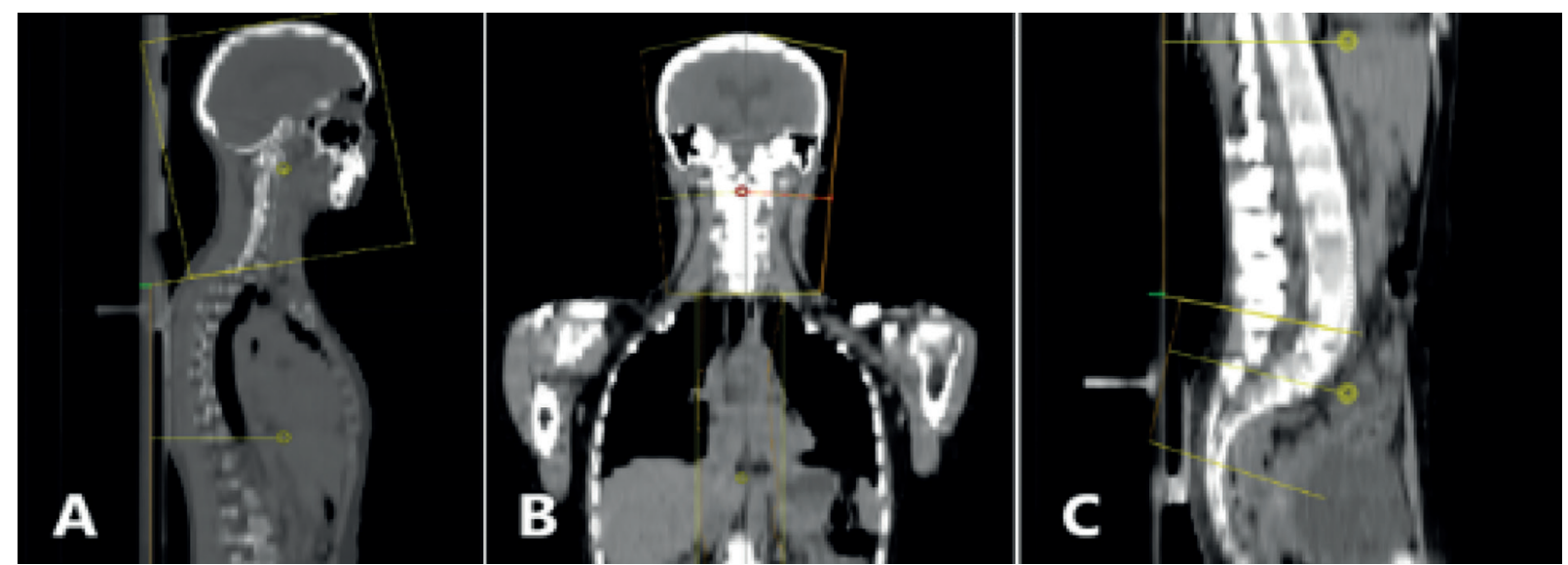

Figure 1. The effect of collimation of treatment fields between the first and the second treatment field - the first junction: $\mathbf{A}$ - lateral view; $\mathbf{B}$ - frontal view. $\mathbf{C}$ - the effect of the changing of treatment table rotation between the first and second treatment field - the second junction (lateral view) 
data collected during the treatment and saved in the oncology information system (Aria). The images ( $\mathrm{kV}$ or PV) used to verify the patient's position during the treatment were audited for all patients. Distances between the positions of the isocentres were collected and checked against the planned distances. Each shift in the direction towards the head was noted as "+ value" and each shift in the direction towards the feet was noted as a negative value compared to the planned distance. Based on that data and the differences between the planned and the treated distances between the positions of isocentres, we created second (actually treated) plans. The planned (P) and treated (T) parameters as dose delivered to particular volume of the PTV (\%), minimum and maximum dose, mean and median total dose, homogeneity index - HI (RTOG) were collected and compared.

\section{Study endpoints}

Overall survival (OS) was evaluated using the Kaplan-Meier method. Progression-free survival was measured from the date of the end of treatment to the date of local or distant progression, or death. The date of death was obtained from the National Cancer Registry. Treatment plans were reviewed in patients with recurrence of the disease, in order to assess the exact location and dose delivered to the relapse site. Toxicity was evaluated based on the RTOG/EORTC criteria [19].

\section{Results}

\section{Group characteristics}

We performed a retrospective analysis of CSI treatment plans of 40 patients ( 27 male and 13 female) with brain tumours (22 medulloblastoma, 10 ependymoma, 5 germinoma, 2 pri- mitive neuroectodermal tumours [PNET], 1 anaplastic oligodendroglioma) treated in our Institution between the years 2005 and 2014. The study group consisted of 14 children and 26 adults. The median age was 21 years at the time of diagnosis (range:4-43). All the patients were treated with curative intent, including those with metastases in the spinal region, which were diagnosed in 4 patients. The mean spine volume was 129 $\mathrm{cm}^{3}$ and the mean spine length was $57 \mathrm{~cm}$.

\section{Fractionation and doses}

Patients were irradiated with a fraction dose (fd) of 1.5-1.8 Gy to the spinal regions and 1.5-2.0 Gy to the brain. All but one received a two phase treatment: in the first phase, the brain and spine were irradiated, in the second, a boost was delivered only to the residual tumour or tumour bed. The median total dose (TD) in the first phase was 36 Gy and the median boost dose was $18 \mathrm{~Gy}$. The PBC algorithm was used in the case of 21 patients, AAA in 19. The mean doses delivered to the organ at risk were within the range of 0.82-6.82Gy for the lenses, 25.33-55.68 Gy for the ears, 13.36-45.26 Gy for the parotids, 3.97-31.27 Gy for the thyroid, 1.26-20.23 Gy for the heart, 1.39-9.90 Gy for the lungs, 0.35-6.18 Gy for the breasts, 2.42-8.85 Gy for the liver, 0.69-14.65 Gy for the bowel, 0.47-3.88 Gy for the kidneys and 0.14-18.9 1Gy for the bladder. Data concerning the delivery of a total number of 902 fractions and 1635 isocentre positions was collected. The planned (P) and treated $(T)$ parameters are presented in table I.

\section{Follow-up}

During the median follow-up (FU) of 58 months, 10 patients died. One-, 2-and 5-year OS was 95\%, 89\% and 78\%, respectively.

Table I. The dosimetric parameters obtained by comparing the original plans and reconstructed dose distribution

\begin{tabular}{|c|c|c|c|}
\hline Parameter & $\%$ diff $\%$ in median dose $(P / T)$ & $\%$ diff $\%$ in mean dose $(\mathrm{P} / \mathrm{T})$ & Range \\
\hline $\mathrm{D}_{70 \%}$ & $-0.02 \%$ & $-0.20 \%$ & $-5.01 \%$ to $+7.62 \%$ \\
\hline $\mathrm{D}_{75 \%}$ & $-0.03 \%$ & $-0.23 \%$ & $-5.91 \%$ to $+7.01 \%$ \\
\hline $\mathrm{D}_{80 \%}$ & $-0.03 \%$ & $-0.47 \%$ & $-6.79 \%$ to $+6.00 \%$ \\
\hline $\mathrm{D}_{85 \%}$ & $-0.06 \%$ & $-1.06 \%$ & $-10.93 \%$ to $+4.60 \%$ \\
\hline $\mathrm{D}_{90 \%}$ & $0.00 \%$ & $-1.22 \%$ & $-11.93 \%$ to $+3.54 \%$ \\
\hline$D_{95 \%}$ & $-0.01 \%$ & $-1.84 \%$ & $-16.27 \%$ to $+3.49 \%$ \\
\hline $\mathrm{D}_{98 \%}$ & $-0.06 \%$ & $-2.43 \%$ & $-25.36 \%$ to $+3.89 \%$ \\
\hline$D_{2 \%}$ & $-0.10 \%$ & $-2.55 \%$ & $-27.75 \%$ to $+3.21 \%$ \\
\hline$D_{\text {min }}$ & $-0.13 \%$ & $-3.73 \%$ & $-30.02 \%$ to $+4.12 \%$ \\
\hline $\mathrm{D}_{\max }$ & $0.04 \%$ & $2.40 \%$ & $-3.03 \%$ to $+26.71 \%$ \\
\hline median $\mathrm{D}_{\mathrm{TD}}$ & $0.00 \%$ & - & $-4.21 \%$ to $8.47 \%$ \\
\hline mean $\mathrm{D}_{\mathrm{TD}}$ & - & $-0.01 \%$ & $-3.67 \%$ to $+9.77 \%$ \\
\hline$H(P)$ & 1.22 & 1.25 & 1.08 to 1.81 \\
\hline$H I(T)$ & 1.25 & 1.28 & 1.08 to 1.87 \\
\hline$D_{\text {max }} / D_{\text {min }}(P)$ & 1.34 & 1.41 & 1.13 to 2.10 \\
\hline$D_{\max } / D_{\min }(T)$ & 1.42 & 1.51 & 1.13 to 2.83 \\
\hline $\mathrm{D}_{\max } / \mathrm{D}_{\min }(\mathrm{P} / \mathrm{T})$ & 0.18 & 0.11 & $-0.05 \%$ to $1.08 \%$ \\
\hline
\end{tabular}

$\%$ diff - (Dose planned-Dose treated)/Dose planned x 100\%; Dose $n$ - dose received by certain percentage of the volume of the PTV; range relates to all mesured plans 
Tumour relapsed in 16 patients - 13 in the brain, 1 in the brain and then in the spine, and 1 in the spine only; 1 patient was diagnosed with multiple bone metastases. Eight patients with relapse died due to the progression of the disease. One-, 2- and 5-year PFS was 89\%, 76\% and 54\%, respectively. Patients with spine relapses had plans with HI of $1.234(P), 1.245$ (T) and 1.154 $(P), 1.193(T)$, respectively. Spine metastases were observed inside the treatment fields, in regions which were covered with a homogenous dose during treatment. No neurologic complications caused by CSI were diagnosed. No radiologic evidence of radiation-induced necrosis was observed.

\section{Conclusions}

So far, this is the first study which evaluated CSI treatment planning techniques with regard to actual everyday treatment delivery implementation, the long-term treatment results and the pattern of progression [20-35]. The technique presented in our study is simple and can be easily implemented - even in departments with limited resources. In some countries, more sophisticated radiotherapy systems, even if potentially available, are not used due to their complexity and the time consuming planning and delivery involved [29-33]. One approach to expand the possibilities of the existing equipment is to install some kind of platform-independent expansions like the ring-based compensator IMRT system proposed by Van Schelt et al. [34]. The other is to refine the planning techniques used, as proposed in our paper to reduce workload during treatment planning and, thanks to the simple irradiation technique, also in-room time which allows for optimization of accelerator use without compromising the quality of the treatment.

Our research is not only a theoretical study dealing with the technical aspects of certain irradiation techniques. Our findings are backed both by dose distribution recalculations based on real-life, everyday position shifts, and by the clinical results of treatment. No symptoms of any spinal cord injury in our patients can be considered a strong confirmation of our theoretical assumptions; this is also supported by calculations of the dose distribution reflecting the real position of the patient.

A limitation of our study is its retrospective character, which makes it impossible to assess whether the positioning data reported in the system are indeed parameters during treatment delivery (if the data was correctly saved in the system) or e.g. the data on the isocenter position after the initial verification because not always the imaging was repeated after position correction. The data concerning dose delivered was re-checked in 6 patients, who, according to the calculations, received less than $80 \%$ of the planned doses. Half of them were planned with the PBC algorithm, half with AAA. In 3 patients "cold spots" were observed in the thoracic-lumbar junction, 2 in the cervical-thoracic junction and 1 in the lumbar part of the spine. The majority of patients were irradiated with good (>80\% of the planned dose in $90 \%$ of the patients) or very good (>90\% of the planned dose in $56 \%$ of the patients) accuracy.
When comparing the groups: the group with less than $<80 \%$ of the planned dose delivered, the group with $80-90 \%$ of the planned dose delivered and the group $>90 \%$ of the planned dose delivered, we observed that patients who had the best results were those with the largest mean and median length of the spine were: in the group $<80 \%$ : mean and median were 63 and $67 \mathrm{~cm}$, in the group 80-90\%: mean and median was $86 \mathrm{~cm}$, and in the group >90\%: mean and median was equal $94 \mathrm{~cm}$. The necessity of sedation of children did not influence the deviations observed in table I.

When discussing the drawbacks of the study, we should also mention the histopathological diagnosis of patients with ependymoma (which is, unless disseminated, no longer an indication for craniospinal irradiation) and 1 patient with PNET (which is no longer recognized according to the new WHO classification). This fact however does not influence the conclusions of the study aimed at the technical aspects of CSI.

SJIG is safe and produces very good long-term outcomes. Treatment planning and delivery is simple and less time consuming than the junction-shift techniques, with good reproduction of the planned dose distribution during actual treatment, assuming that image guidance is available and used on a daily basis.

\section{Conflict of interest: none declared}

\section{Aleksandra Napieralska}

Maria Sklodowska-Curie National Research Institute of Oncology Gliwice Branch

Radiotherapy Department

Wybrzeże Armii Krajowej 15

44-101 Gliwice, Poland

e-mail:olanapieralska@gmail.com

Received: 23 Apr 2021

Accepted: 15 Jun 2021

\section{References}

1. Athiyaman $\mathrm{H}$, Mayilvaganan A, Singh D. A simple planning technique of craniospinal irradiation in the eclipse treatment planning system. J Med Phys. 2014; 39(4): 251-258, doi: 10.4103/0971-6203.144495, indexed in Pubmed: 25525314.

2. Jenkin D. The radiation treatment of medulloblastoma. J Neurooncol. 1996; 29(1): 45-54, doi: 10.1007/BF00165517, indexed in Pubmed: 8817415.

3. Wang $Z$, Jiang W, Feng $Y$, et al. A simple approach of three-isocenter IMRT planning for craniospinal irradiation. Radiat Oncol. 2013; 8: 217, doi: 10.1186/1748-717X-8-217, indexed in Pubmed: 24044521.

4. Zong-Wen S, Shuang-Yan Y, Feng-Lei Du, et al. Radiotherapy for Adult Medulloblastoma: Evaluation of Helical Tomotherapy, Volumetric Intensity Modulated Arc Therapy, and Three-Dimensional Conformal Radiotherapy and the Results of Helical Tomotherapy Therapy. Biomed Res Int. 2018; 2018: 9153496, doi: 10.1155/2018/9153496, indexed in Pubmed: 29750173.

5. Wang K, Meng H, Chen J, et al. Plan quality and robustness in field junction region for craniospinal irradiation with VMAT. Phys Med. 2018; 48: 21-26, doi: 10.1016/j.ejmp.2018.03.007, indexed in Pubmed: 29728225.

6. Seravalli E, Bosman M, Lassen-Ramshad Y, et al. Dosimetric comparison of five different techniques for craniospinal irradiation across 15 European centers: analysis on behalf of the SIOP-E-BTG (radiotherapy working group). Acta Oncol. 2018; 57(9): 1240-1249, doi: 10.1080/0284186X.2018.1465588, indexed in Pubmed: 29698060. 
7. Sarkar B, Munshi A, Manikandan A, et al. A low gradient junction technique of craniospinal irradiation using volumetric-modulated arc therapy and its advantages over the conventional therapy. Cancer Radiother. 2018; 22(1):62-72, doi: 10.1016/j.canrad.2017.07.047, indexed in Pubmed: 29195796.

8. Studenski MT, Shen X, Yu Y, et al. Intensity-modulated radiation therapy and volumetric-modulated arc therapy for adult craniospinal irradiation--a comparison with traditional techniques. Med Dosim. 2013; 38(1): 48-54, doi: 10.1016/j.meddos.2012.05.006, indexed in Pubmed: 22878118.

9. Ahmed SK, Kruse JJ, Bradley TB, et al. Clinical efficacy and safety of a highly conformal, supine, hybrid forward and inverse planned intensity modulated radiation therapy technique for craniospinal irradiation. Acta Oncol. 2018;57(5):629-636, doi: 10.1080/0284186X.2017.1400686, indexed in Pubmed: 29129125.

10. Srivastava R, Saini G, Sharma PK, et al. A technique to reduce low dose region for craniospinal irradiation (CSI) with RapidArc and its dosimetric comparison with 3D conformal technique (3DCRT). J Cancer Res Ther. 2015; 11(2): 488-491, doi: 10.4103/0973-1482.144556, indexed in Pubmed: 26148626.

11. Bandurska-Luque A, Piotrowski T, Skrobała A, et al. Prospective study on dosimetric comparison of helical tomotherapy and 3DCRT for craniospinal irradiation - A single institution experience. Rep Pract Oncol Radiother. 2015; 20(2): 145-152, doi: 10.1016/j.rpor.2014.12.002, indexed in Pubmed: 25859405

12. Myers PA, Mavroidis P, Papanikolaou N, et al. Comparing conformal, arc radiotherapy and helical tomotherapy in craniospinal irradiation planning. J Appl Clin Med Phys. 2014; 15(5): 4724, doi: 10.1120/jacmp. v15i5.4724, indexed in Pubmed: 25207562.

13. Parker WA, Freeman CR. A simple technique for craniospinal radiotherapy in the supine position. Radiother Oncol. 2006; 78(2): 217-222, doi: 10.1016/j.radonc.2005.11.009, indexed in Pubmed: 16330119.

14. Hadley A, Ding GX. A single-gradient junction technique to replace multiple-junction shifts for craniospinal irradiation treatment. Med Dosim. 2014; 39(4): 314-319, doi: 10.1016/j.meddos.2014.05.004, indexed in Pubmed: 24913466.

15. Cao F, Ramaseshan R, Corns R, et al. A three-isocenter jagged-junction IMRT approach for craniospinal irradiation without beam edge matching for field junctions. Int J Radiat Oncol Biol Phys. 2012; 84(3): 648654, doi: 10.1016/j.ijrobp.2012.01.010, indexed in Pubmed: 22436794.

16. Stoker JB, Grant J, Zhu XR, et al. Intensity modulated proton therapy for craniospinal irradiation: organ-at-risk exposure and a low-gradient junctioning technique. Int J Radiat Oncol Biol Phys. 2014; 90(3): 637644, doi: 10.1016/j.jirobp.2014.07.003, indexed in Pubmed: 25194667.

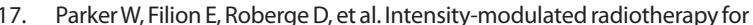
craniospinal irradiation: target volume considerations, dose constraints, and competing risks. Int J Radiat Oncol Biol Phys. 2007; 69(1): 251-257, doi: 10.1016/j.jjrobp.2007.04.052, indexed in Pubmed: 17707279.

18. Kusters JM, Louwe RJW, van Kollenburg PGM, et al. Optimal normal tissue sparing in craniospinal axis irradiation using IMRT with daily intrafractionally modulated junction(s). Int J Radiat Oncol Biol Phys. 2011; 81(5): 1405-1414, doi: 10.1016/j.jijrobp.2010.07.1987, indexed in Pubmed: 21300472.

19. Cox JD, Stetz J, Pajak TF. Toxicity criteria of the Radiation Therapy Oncology Group (RTOG) and the European Organization for Research and Treatment of Cancer (EORTC). Int J Radiat Oncol Biol Phys. 1995; 31(5): 1341-1346, doi: 10.1016/0360-3016(95)00060-C, indexed in Pubmed: 7713792.

20. Mah K, Danjoux CE, Manship S, et al. Computed tomographic simulation of craniospinal fields in pediatric patients: improved treatment accuracy and patient comfort. Int J Radiat Oncol Biol Phys. 1998; 41 (5): 997-1003, doi: 10.1016/s0360-3016(98)00108-4, indexed in Pubmed: 9719108.

21. Phillips C, Willis D, Cramb J, et al. A modified technique for craniospina irradiation in children designed to reduce acute and late radiation toxicity. Australas Radiol. 2004; 48(2): 188-194, doi: 10.1111/j.1440-1673.2004.01295.x, indexed in Pubmed: 15230753.

22. South M, Chiu JK, Teh BS, et al. Supine craniospinal irradiation using intrafractional junction shifts and field-in-field dose shaping: early experience at Methodist Hospital. Int J Radiat Oncol Biol Phys. 2008; 71(2): 477-483, doi: 10.1016/j.jijrobp.2007.10.029, indexed in Pubmed: 18164864.

23. Hawkins RB. A simple method of radiation treatment of craniospinal fields with patient supine. Int J Radiat Oncol Biol Phys. 2001; 49(1): 261-264, doi: 10.1016/s0360-3016(00)01367-5, indexed in Pubmed: 11163523

24. Christ G, Denninger D, Dohm OS, et al. Craniospinal radiotherapy in an advanced technique. Strahlenther Onkol. 2008; 184(10): 530-535, doi: 10.1007/s00066-008-1843-1, indexed in Pubmed: 19016043.

25. Strojnik A, Méndez I, Peterlin P. Reducing the dosimetric impact of positional errors in field junctions for craniospinal irradiation using VMAT. Rep Pract Oncol Radiother. 2016; 21(3): 232]239, doi: 10.1016/j. rpor.2016.03.002, indexed in Pubmed: 27601956.

26. Sharma DS, Gupta T, Jalali R, et al. High-precision radiotherapy for craniospinal irradiation: evaluation of three-dimensional conformal radiotherapy, intensity-modulated radiation therapy and helical TomoTherapy. Br J Radiol. 2009; 82(984): 1000-1009, doi: 10.1259/ bjr/13776022, indexed in Pubmed: 19581313.

27. Lee YK, Brooks CJ, Bedford JL, et al. Development and evaluation of multiple isocentric volumetric modulated arc therapy technique for craniospinal axis radiotherapy planning. Int J Radiat Oncol Biol Phys. 2012; 82(2): 1006-1012, doi: 10.1016/j.ijrobp.2010.12.033, indexed in Pubmed: 21345612.

28. Grover S, Gudi S, Gandhi AK, et al. Radiation Oncology in India: Challenges and Opportunities. Semin Radiat Oncol. 2017; 27(2): 158-163, doi: 10.1016/j.semradonc.2016.11.007, indexed in Pubmed: 28325242.

29. Barton $\mathrm{MB}$, Zubizarreta EH, Polo Rubio JA. Radiotherapy in smal countries. Cancer Epidemiol. 2017; 50(Pt B): 257-259, doi: 10.1016/j. canep.2017.06.007, indexed in Pubmed: 29120833.

30. Zubizarreta EH, Fidarova E, Healy B, et al. Need for radiotherapy in low and middle income countries - the silent crisis continues. Clin Oncol (R Coll Radiol). 2015; 27(2): 107-114, doi: 10.1016/j.clon.2014.10.006, indexed in Pubmed: 25455407.

31. Zubizarreta E, Van Dyk J, Lievens Y. Analysis of Global Radiotherapy Needs and Costs by Geographic Region and Income Level. Clin Oncol (R Coll Radiol). 2017; 29(2): 84-92, doi: 10.1016/j.clon.2016.11.011, indexed in Pubmed: 27939337.

32. Donkor A, Luckett T, Aranda S, et al. Barriers and facilitators to implementation of cancer treatment and palliative care strategies in lowand middle-income countries: systematic review. Int J Public Health. 2018; 63(9): 1047-1057, doi: 10.1007/s00038-018-1142-2, indexed in Pubmed: 29974131.

33. Van Schelt J, Smith DL, Fong N, et al. A ring-based compensator IMRT system optimized for low- and middle-income countries: Design and treatment planning study. Med Phys. 2018; 45(7): 3275-3286, doi: 10.1002/mp.12985, indexed in Pubmed: 29777595.

34. Holmes JA, Chera BS, Brenner DJ, et al. Estimating the excess lifetime risk of radiation induced secondary malignancy (SMN) in pediatric patients treated with craniospinal irradiation (CSI): Conventional radiation therapy versus helical intensity modulated radiation therapy. Pract Radiat Oncol. 2017; 7(1): 35-41, doi: 10.1016/j.prro.2016.07.002, indexed in Pubmed: 27663930 